\title{
When Technology Encounters Culture: A Closer Watch on Dajia Mazu Patrol and Pilgrimage in Taiwan
}

\author{
Ching-Yun Mu ${ }^{1}$, Will Y. Lin ${ }^{1}$, Zheng-Yu Chen ${ }^{1}$, Tien-Yin Chou ${ }^{1}$, Lung-Shih Yang ${ }^{2}$, Cheng-Shu Kao ${ }^{3}$ \\ ${ }^{1}$ GIS Research Center, Feng Chia University, Taichung, Chinese Taipei \\ ${ }^{2}$ Vice President's Office, Feng Chia University, Taichung, Chinese Taipei \\ ${ }^{3}$ Vice Chairman Room, Feng Chia University, Taichung, Chinese Taipei \\ Email: william@gis.tw, will.dwu@gmail.com
}

Received October 29, 2011; revised December 25, 2011; accepted January 8, 2012

\begin{abstract}
Mazu, a protective sea God, has been worshipped by residents of Taiwan and southeast coast of mainland China as well as overseas Chinese around the globe for hundreds of years. The number of people around the world under her influence of religious belief and moral culture can reach up hundreds of million. Every year in lunar March, the overone-week long Mazu patrol and pilgrimage held by Jenn Lann Temple in Dajia County of Taiwan attracts millions of pilgrims and tourists around the world to participate in one of the biggest religious events in the world. To keep track of the entire patrol and pilgrimage, Jenn Lann Temple has been cooperating with GIS Research Center, Feng-Chia University since 2008, setting up a GPS receiver, a digital camera, and 4 video recorders on Mazu's palanquin. Both real-time position of the palanquin and live videos on the scene along the way of pilgrimage were published on the Internet, providing pilgrims, tourists and viewers around the world with an open access to observe the entire event. This paper details this initiative of introducing spatial technology to large cultural events. The study collects the historic tracks of Mazu's palanquin during the pilgrimage from 2008 to 2010, analyzes their spatial-temporal attributes, and elicits several interesting facts behind the figures and maps. It also explores how spatial technologies can help organize largescale events and even accelerate the dissemination of culture.
\end{abstract}

Keywords: Mazu Culture; Pilgrimage; GPS; WebGIS

\section{Introduction}

Mazu is the sea goddess who has been worshipped by Taiwan residents and overseas Chinese for centuries. Since the Ming and Qing Dynasties, the Mazu belief has been the most popular folk belief in Taiwan, where more than 760 Mazu temples of all scales were built [1-3]. 39 of them can be found in historical records, including the Dajia Jenn Lann Temple in Taichung [4,5]. The most famous event of Jenn Lann Temple, Mazu Patrol and Pilgrimage, can date back to the late 18th century when the temple was rebuilt, and every 12 years pilgrims sailed with Mazu statue to Mei Chou, China where the statue originated from [6,7]. Since 1990's, Mazu Patrol and Pilgrimage every lunar March, lasting for 8 to 9 days and gathering more than hundreds of thousands of pilgrims, has recently been recognized by Discovery Channel as one of three major religious festivals around the world [8], along with the Muslim Pilgrimage to Mecca and Hindu Rite of Bathing in the Ganges River [1].

With the increasing number of temples that join in the patrol, the festival scale expands year by year. In order for most pilgrims and tourists to seize the best timing to worship the Mazu palanquin or observe the pilgrimage, since year of 2008 Dajia Jenn Lann Temple has been cooperating with GIS Research Center, Feng Chia University setting up a GPS receiver, four digital cameras, and a digital video recorder (DVR) on the palanquin to record its real-time position and live videos from its angles of view. The positions and videos are then published on the internet for the public to access. This initiative can also assist palanquin carriers to control patrol speed, and facilitate the preparation for food, drinks, and even accommodation by staff of temples that join in the festival.

Publishing the real-time position palanquin on the internet can benefit pilgrims, tourists and festival preparation staff along the patrol and pilgrimage routes [9]. The historic trajectories can also help festival organizers decide the optimal routes, and re-examine the planning in hindsight to do better on next year's festival [10]. This article will detail the initiative of introducing spatial technologies to a large cultural event, which includes the positioning technology, equipment and operation framework adopted by the initiative. It also explores how spatial technologies can help organize the large-scale event 
and even accelerate the spread of culture.

\section{History of Mazu Patrol and Pilgrimage}

From her earliest stage as a folk belief hundreds of years ago, Mazu's influence has grown in its breadth and intensity on populations and cultures in Taiwan, coastal China, and Southeast Asia. Many scholars have named it "Mazu culture" in recent years [2,10]. Mazu's life stories, historical records and related research have been written in last decades. This section only briefly describes some facts related to pilgrimage routes, and three folk activities related to palanquin position, including Patrol and Pilgrimage, Crawling under the Palanquin, and Hijacking the Palanquin. In the following sections, the article will describe how spatial science and technology can benefit these activities.

\subsection{Patrol and Pilgrimage}

In the early days, most of the Mazu's statues in the Mazu temples of Taiwan which came with Mainland Chinese immigrants had "division of spiritual power" from the Mazu temples of their hometowns [11]. The so-called "division of spiritual power" means that believers or even temples ask for the Mazu's statues or incense from some "effective" temples [7,12]. After these incense or statues with division of spiritual power arrived in Taiwan safely with believers, they were normally honored in the place where believers first landed or settled down [13]. Afterwards, when believers lived a more stable life with better financial ability, they called on other believers to raise funds for the construction of Mazu temples. BeiKang Mazu Temple, Lu-Gang Tian Hou Temple, BenGang Gang Kou Temple, An-Ping Tian Hou Mazu Temple, Lu-Erh-Men Tin Hau Temple, Shin-Gang Feng Tien Temple are those Mazu temples which were built in the early stages [6]. Later, with the increasing number of Mazu's believers, Mazu temples of all sizes have been continually built all around Taiwan. These Mazu temples at the late stage would also ask for the avatar of the Mazu's statue by the way of division of spiritual power from the above-mentioned Mazu temples which have longer history or a larger scale. Generally, believers believe that the spiritual power of the "ancestor temple" is stronger than the division of spiritual power of the "child temple". Hence, the child temple needs to go back to the ancestor temple to process the ritual of "division of incense fire" in order to keep the spiritual power unflagging and simultaneously verify the kinship of incense fire between the ancestor temple and the subsidiary templethis is how "pilgrimage" comes from [11].

Dajia Mazu obtained division of spiritual power from Mei-Zhou Mazu Temple during the Yongzheng period of Qing Dynasty. Since Dajia Jenn Lann Temple was built during the Qianlong period of Qing Dynasty, around every twelve years Dajia Jenn Lann Temple where Dajia Mazu is located has been going to Mei-Zhou Mazu Temple for paying respect to ancestors and offering incense in the name of pilgrimage. After Taiwan was politically separated from China, people started offering incense to Bei-Kang Chao Tian Mazu Temple annually instead, and each pilgrimage lasts for eight days and seven nights, which is known as "patrol and pilgrimage" $[1,5,6]$. The so-called "patrol" means that Mazu goes on an inspection tour around the area under her control while "pilgrimage" means that believes invite Mazu to visit temples in other regions. The patrol team consists of people from different social classes who mainly participate in the patrol in order to thank the Goddess for answering their wishes and to pray for blessings. The patrol crosses over four counties including Taichung, Changhua, Yunlin, and Chiayi, more than twenty villages and towns, and more than eighty temples. The walking distance is about three hundred kilometers. Since 1988, the patrol has become a round-trip interview with Shin-Gang Feng Tien Temple and the activity also extends into nine days and eight nights due to the increasing number of believers and temples $[2,6,8]$.

During the patrol, the team of the palanquin follows the route which is arranged in advance. When the team passes residential areas, stores and temples, people along the way worship with incense in their hands in order to pray for Mazu's blessing [12]. Domestic and international tourists who come to visit because of respect for the fame also wait for Mazu's palanquin to pass on the route which is announced by the temple. However, the movement speed of the pilgrimage is greatly affected by enthusiastic people on the way; therefore, the arranged schedule of the pilgrimage is usually delayed. The time which the palanquin arrives at each hosting temple varies greatly. Although for believers waiting patiently for the arrival of Mazu's palanquin is a kind of test to see whether they have strong faith in Goddess, for tourists, waiting long in the crowded crowd or even missing Mazu's palanquin is torturing. Therefore, the real-time location of Mazu's palanquin is fairly crucial reference information for tourists and pilgrims who participate in the celebration.

\subsection{Crawling under the Mazu's Palanquin}

When the palanquin passes by, believers not only worship with burning joss-sticks, but also lie down or kneel down on the moving route, allowing the palanquin to cross over their backs, which is called "crawling under Mazu's palanquin". It obtains the meanings of thanking the Goddess and praying for peace. The custom of crawling under Mazu's palanquin exists almost in temples of different sizes everywhere and participants are of 
different genders and different ages [12]. People deeply believe that the Mazu's Gold Body is sitting in the palanquin. If Mazu's statue crosses over people or people crawl under the palanquin, disasters can be eliminated and people can pray for peace and blessing. Believers need to wait on the route which Mazu's palanquin will pass in advance and show their respect by lying down and kneeling down once the palanquin comes close [2,7]; thus, the intended moving route of Mazu's palanquin and the immediate location during the patrol are even more important for believers.

\subsection{Palanquin-Robbing}

Most local believers believe that Mazu will bless the place where Mazu's palanquin has visited; thus, they all hope that their residence or business locations can be on the route of the patrol and pilgrimage [8]. However, there is a time constraint on the patrol and pilgrimage, meaning that the patrol can only pass through a limited number of temples or regions. Hence, every year the phenomenon of "robbing Mazu's palanquin" normally occurs during the patrol. The action of robbing Mazu's palanquin is mainly dominated by local factions and local religious elites, who not only attempt to receive Mazu's blessing, but also strive to extend their organization's sphere of influence. Robbing Mazu's palanquin becomes the battle for influence among local religious elites from each region [8]. The occurrence of rubbing the palanquin and the extended time are unpredictable; therefore, whenever the palanquin is rubbed, it is unable to follow the arranged route and schedule. A larger scale action of Mazu's palanquin may even influence the next temple which hosts the Goddess in arranging accommodation. At this time, controlling the location of the palanquin and estimating its arrival time will be of great help for the temple staff who hosts the Goddess' patrol to make arrangements.

\section{Application of Spatial Technologies}

The previous section describes the origin and form of Patrol and Pilgrimage, and states how real-time position and historical trajectory of the palanquin can benefit the festival organizers, temple staff, pilgrims, and tourists [9]. This section further introduces how to achieve those benefits by applying spatial technologies, including Global Positioning System (GPS) and web-based geographic information system (WebGIS), followed by the description of system architecture and operation mechanism.

\subsection{Global Positioning System (GPS)}

Broadly speaking, global positioning refers to the technology that uses satellites and positioning algorithms to determine coordinates of objects on the surface of the earth, also known as Global Navigation Satellite System (GNSS). Global Positioning System (GPS) is one of four current positioning systems under commercial operation, along with Global Orbiting Navigation Satellite System (GLONASS) developed by Russian, Galileo satellite navigation system (Galileo) by Europe and Compass Navigation Satellite System (CNSS) by China.

Among them, however, GPS has the highest commercial popularity, and become the synonym of global positioning to the public. GPS, developed by United States, consists of 24 positioning satellites, and uses radio signals transmitted by satellite to measure the distance between the satellite and the receiver. In general, it requires at least three satellites for signal receivers to determine the location and altitude of any object on surface of the earth with the accuracy of 10 meters. GPS technology has been widely used in vehicle navigation, fleet monitoring and other commercial uses. As mobile computing and wireless technologies become more and more mature in recent years, GPS has built-in mobile devices such as navigators, mobile phones, PDAs, and car kits.

In this festival, we set up a car kit with a built-in GPS receiver and wireless transmission module on Mazu's palanquin. The car kit will regularly transmit the position data calculated by the GPS receiver back to a centralized control center via wireless connection, which enables to publish the real-time location of the palanquin on a web-based map platform. Pilgrims, tourists, and temple staff thus can have ready access to palanquin's real-time location through a web browser, either on the desktop, laptop, or on mobile devices.

\subsection{Web-Based GIS}

GIS is a multidisciplinary science that combines geography, surveying, cartography and information technology. It uses overlay of map layers and geospatial database to present the map features and attributes on computer screens. The concept of GIS is illustrated in Figure 1. Web-based GIS, simply speaking, is a GIS that publishes map data through the internet or networks [14]. The map data can thus be accessed via web browsers. The abovementioned web-based map platform to publish palanquin's locations is also a WebGIS.

WebGIS is mainly composed of two parts. One part is the user interface, which allows users to browse the map on your web browser, and perform spatial queries of any kind. The other is the GIS server, which is responsible for publishing map data and responding to user's queries or other requests. Compared to traditional stand-alone GIS, WebGIS can be used simultaneously by multiple users. They only need a web browser to access map data. Its highly usability and accessibility makes WebGIS suitable for publishing map data to the public. Map data 


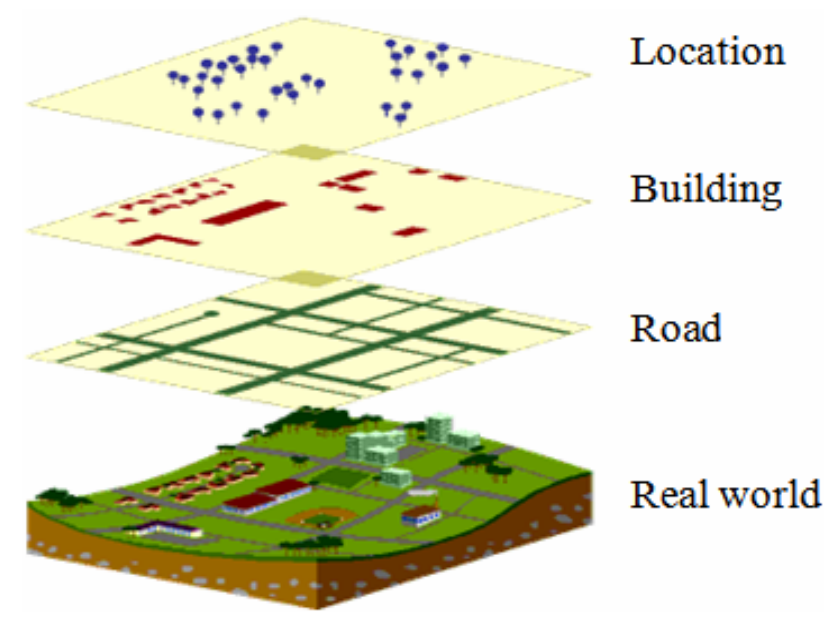

Figure 1. The concept of GIS.

published by WebGIS can also be accessed from mobile devices, which facilitates the access by festival participants using PDAs, cellphones, or other mobile devices.

\subsection{System Architecture and Design}

The entire system architecture can be divided into two parts: the hardware equipped on the palanquin, and the software on the centralized control center, as shown in Figure 2. The upper part of the figure illustrates the hardware devices consisting of one digital camera, four digital video recorders (DVR) with a GPS antenna and a hard disk, and a Car kit with a GPS receiver and a GPRS communication module.

The car kit transmits geographic coordinates of the palanquin fed by the GPS receiver every 30 seconds as well as other necessary information such as speed, bearing, and status back to the centralized control center via GPRS module. The WebGIS server in the control center, to be introduced later, analyzes the coordinates and renders an icon of palanquin on the digital map according to the coordinates. Real-time videos captured by the DVRs setup at four corners of the palanquin are stored in the hard disk. Then, 16 frames within a 2-minute interval of each video are selected and sent to the control center via GPRS module every 2 minutes. Figure 3 is the photo showing the installment of hardware on the back of Mazu's palanquin described above.

The lower part of Figure 2 summarizes a collection of three servers responsible for different software services. The WebGIS server is the Microsoft web server, or Internet Information Server (IIS), where the portal embedded with a web-based map platform is located. The map platform is EasyMap, a product developed by GIS Research Center, Feng Chia University using ASP.NET web programming, which provides map data and application programming interfaces (API) to manipulate and display map features.

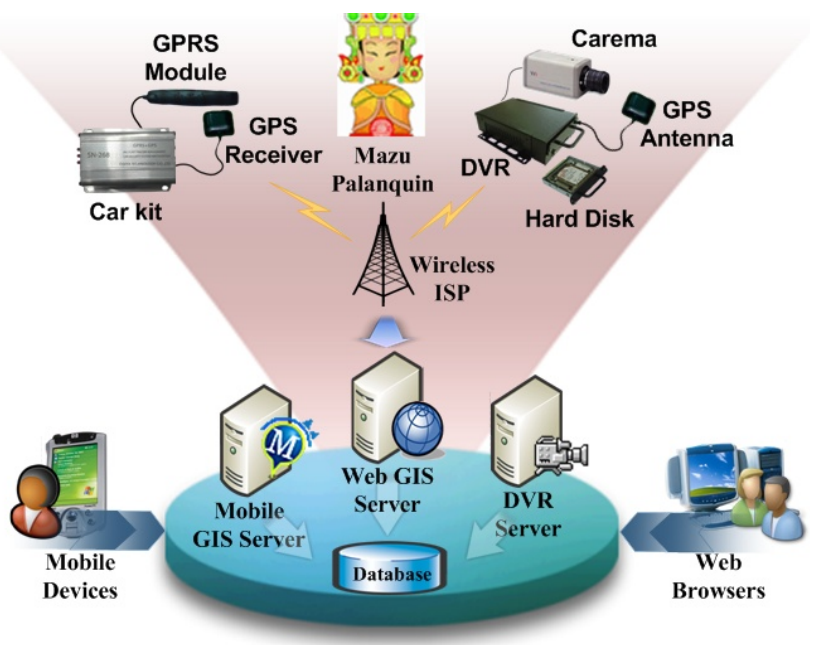

Figure 2. System architecture.

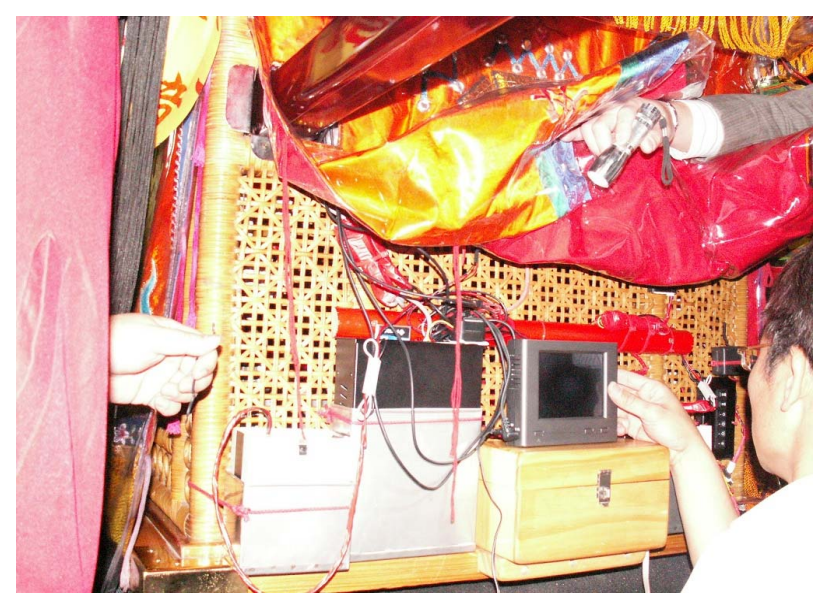

Figure 3. Hardware installment.

DVR server is responsible for managing video data sent by those four DVRs. The selected video frames are then embedded in the portal as well as in mobile portal, which is prepared by Mobile GIS Server separated from WebGIS server to down size of system loading.

Since 2011, the development team offered both i-Phone App and Android App for the public to download and access the mobile portal by smartphones. Pilgrims and tourists who have smartphones can download these free applications from Apple's App Store and Google Android Market, respectively. These smartphone applications can display the palanquin's location in Google Map, instead of EasyMap, and can be used by PDAs and desktop web browsers. It also provides historic trails of Patrol and Pilgrimage on previous days as well as the real-time video with high resolutions. With these applications on mobile clients published to the public in 2011, the number of visits to our services dramatically reached up the summit since 2008, which also reveals that mobile devices could play an important role in diffusion of dailylife technologies. 
From several informal interviews to pilgrims and tourists using our mobile clients, we learned that our services could provide not only the guidance of pilgrimage and patrol, but also a guidebook of tourism in those areas along the route. Staff from Jenn Lann Temple also used the mobile clients for coordination, where the accurate position of palanquin helped with oral communication as well as estimation of time left to prepare for some measurements before the palanquin arrived.

\section{Statistical Analysis}

Data transmitted by the GPS car kit every 30 seconds contain latitude and longitude coordinates and transmission time. The accumulation of data in the database is rapid and tremendous. For example in 2010, there were 11,526 valid records stored in the database. However, those spatial-temporal data can be an important source for statistical analysis, and thus reveal some cultural meanings behind as well as provide a reference for festival organizers to make decisions. This section presents several statistics collected and analyzed from the past three years, including patrol and pilgrimage route, mileage, idling time, and discusses some important issues from cultural and administrative perspectives.

\subsection{Patrol and Pilgrimage Route}

According to literature, the planning of patrol and pilgrimage route depends on several key factors, such as the serving temples, traffic concerns, and non-repetition $[5,7$, 12]. As mentioned earlier, purpose of the patrol is for Mazu to inspect the area under her control; thus those temples within the area naturally taken into account of patrol route. However, temples that participate in the pilgrimage may vary from year to year. For example, in 2010, Jenn Lann Temple decided to expand the pilgrimage, and threw "divination blocks" to ask for Mazu's permission for additional day of pilgrimage, which resulted in as many as 27 temples to participate in the festival for their very first time.

Cross-country routes, whose main consideration is the convenience of traffic and accommodation, are generally along with provincial road number 1 of Taiwan, which is wide and roughly straight, while routes within the township adhere to the principle of non-repetition, in order for palanquin to meet the demand for more local believers. In addition, the forward pilgrimage is subject to arrive in the destination for the scheduled ceremony, so the route on forward trip within the same area often shorter than the backward trip. Figure $\mathbf{4}$ is the route of patrol and pilgrimage where red line indicates the forward trip, much shorter than green backward trip. Figures 5 and $\mathbf{6}$, show both forward and back routes in both Changhua and Yunlin County in 2010, respectively, indicating the principle of non-repetition within a township.

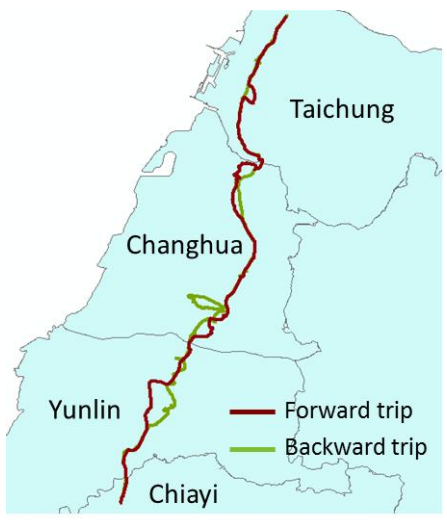

Figure 4. Entire patrol \& pilgrimage routes in 2010.

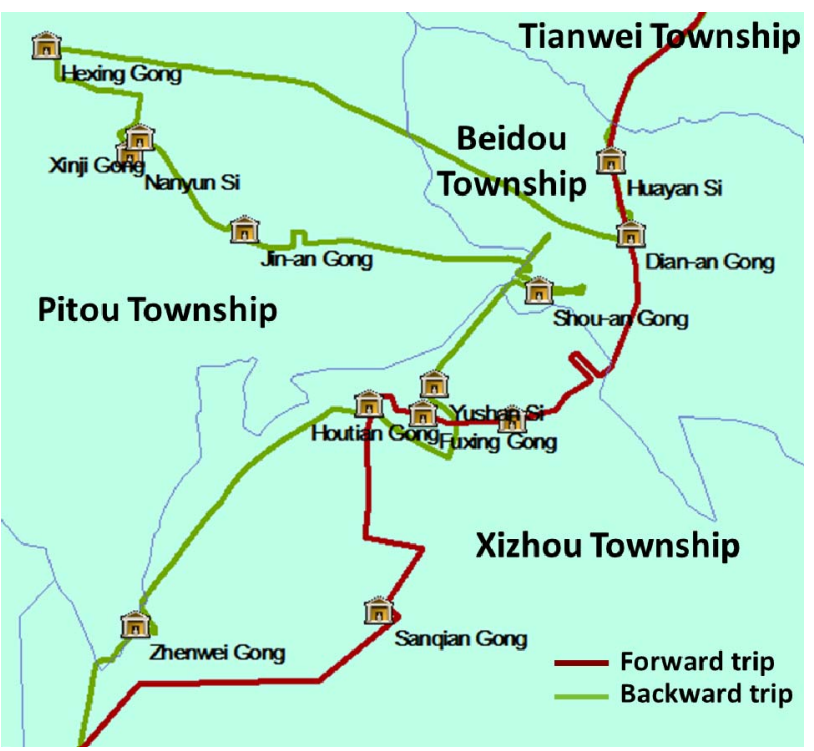

Figure 5. Pilgrimage routes in Changhua County.

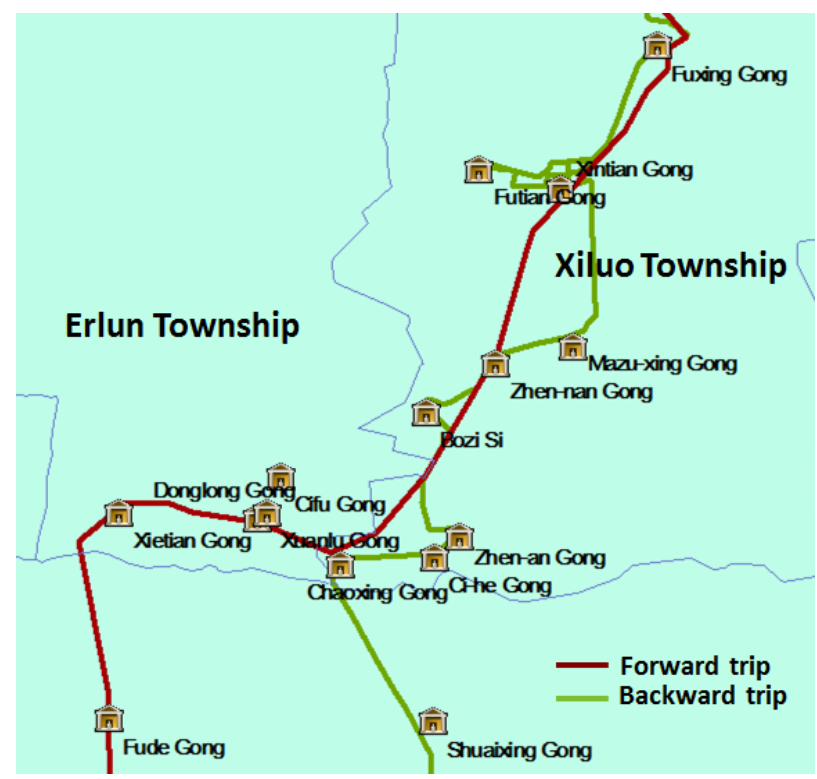

Figure 6. Pilgrimage routes in Yunlin County. 


\subsection{Walking Distance}

Total walking mileages of patrol and pilgrimage from 2008 to 2010 can be obtained by linking consecutive positions of the palanquin, as show in Figure 7. The total walking mileages roughly range from 295 to $300 \mathrm{~km}$, where the forward trips by and larger are 15 to $30 \mathrm{~km}$ longer than the backward trips. That's due to the forward trip subject to a fixed arrival time for scheduled ceremonies as mentioned earlier [12].

In 2010 when 27 more temples joined in the pilgrimage and one more day was added, however, the total walking distance didn't significantly increase; instead the palanquin spent more time on temples along the route and slowed down the speed on the backward trip. This arrangement can be found in Figure 8 where daily walking distances for three years are plotted.

Figure 8 also reveals a fact that the forward trip is one or two days less than the backward trip. The forward trips are always 3 days long while the backward trips are 4 days in 2008 and 2009, but 5 days in 2010. The longest daily walking distance falls in the second day of the forward trip for three years, with a mileage up to $58 \mathrm{~km}$ within a walking period of 18 hours. That's because on the second of patrol the palanquin always passes Chunghwa

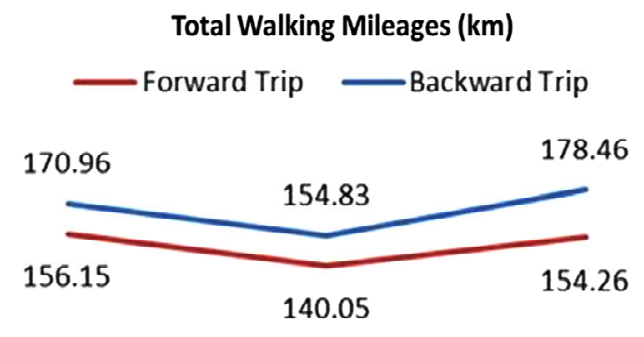

2008

2009

2010 Year

Figure 7. Total mileages of pilgrimage from 2008 to 2010.

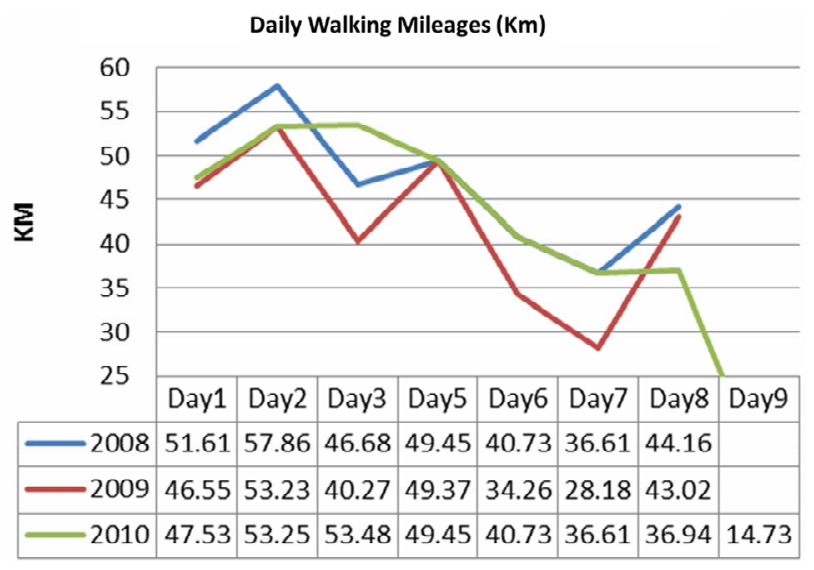

Figure 8. Daily mileages of pilgrimage from 2008 to 2010.
County where around 40 temples join in the patrol, accounting for about one third of the total participating temples. The palanquin doesn't move on day 4 because of ceremonies, and thus the second longest walking distance falls in the first day of backward trip, or day 5 for the entire journey probably because the pilgrims gain more energy from resting on day 4 .

\subsection{Idling Time}

Factors of affecting the idling time of palanquin at a certain temple includes the fame and scale of temple, the relations between the temple and Jenn Lann Temple, the enthusiasm of the people along the way, and "Robbing Mazu's palanquin".

Idling time can be divided into three clusters with different length groups. The first cluster of idling time is 2 to 3 hours, followed by the second cluster of 30 minutes and the third, less than 20 minutes. Temples with long idling time are those sites with high potential to become spots of tourism.

\subsection{Website Analytics}

Estimates of pilgrim and tourist number by the press, governments, or Jenn Lann Temple could range from hundreds of thousands to millions depending on the methods of estimation. However, the trend of increase in popularity of the event since its early years is obvious. The promotion by Jenn Lann Temple in recent years is generally acknowledged as the major driving force to this tendency. Adoption of information technologies is one of measurements used by the temple authority.

Since 2008 when the reporting services of Mazu's palanquin was presented to the public for the first time, the development team has adopted Google Analytics, as shown in Figure 9, as the tool of monitoring the website traffic to filter malicious attacks as well as to analyze the geographic distribution of portal visitors.

According to summaries of Google Analytics on our portal, the average year-by-year increase rate of portal visitor numbers during the festival from 2008 to 2011 is $16.46 \%$. With the provision of smartphone applications in 2011 , however, it dramatically increased by $33.77 \%$ compared with the year of 2010 . Figure 10 shows the distribution of mobile devices used by visitors to access the mobile portal. Android had almost twice as many users as iPhone among our portal visitors. Both major mobile devices accounted for about $85 \%$ of visits.

With those analytics being monitored every year, we can trace the portal visitors, where they are from or how long they stay on the site, and thus estimate the growth of event popularity or even the level of culture dissemination. Figure 11 illustrates the geographic distribution of visitors by cities around the globe in 2011. Over 180 thou- 


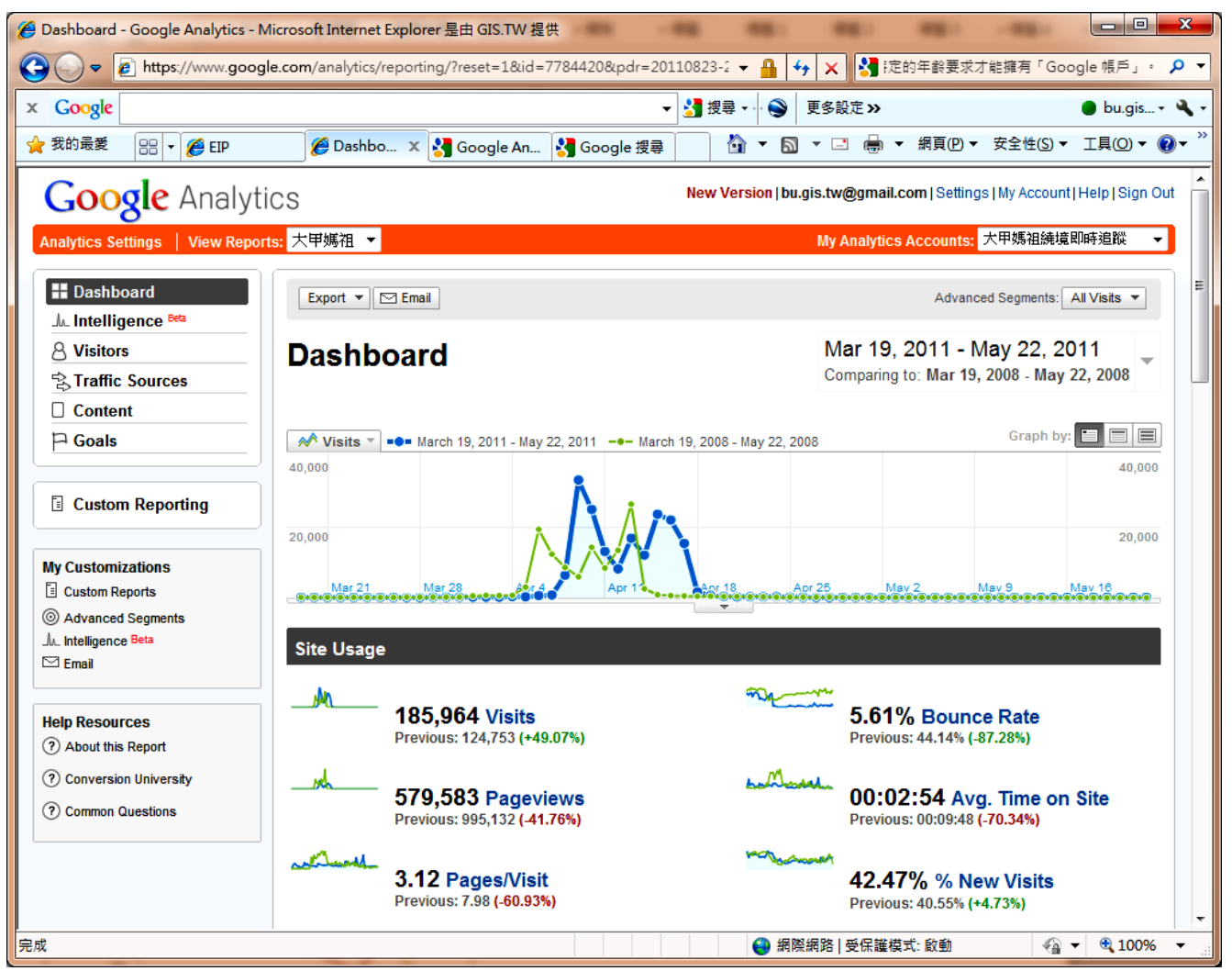

Figure 9. Website Traffic of portal by Google Analytics.

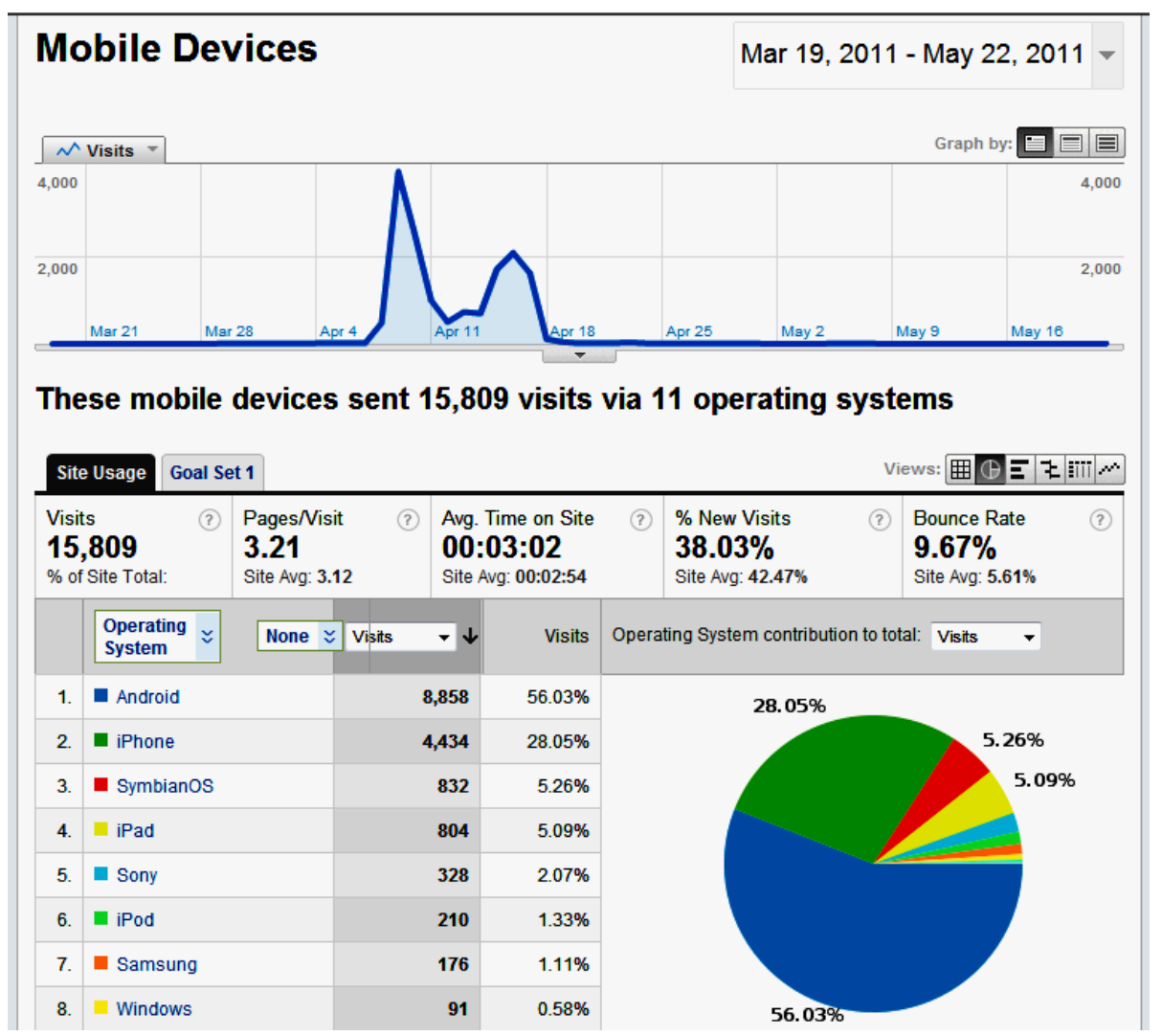

Figure 10. Distribution of visitors by mobile devices. 


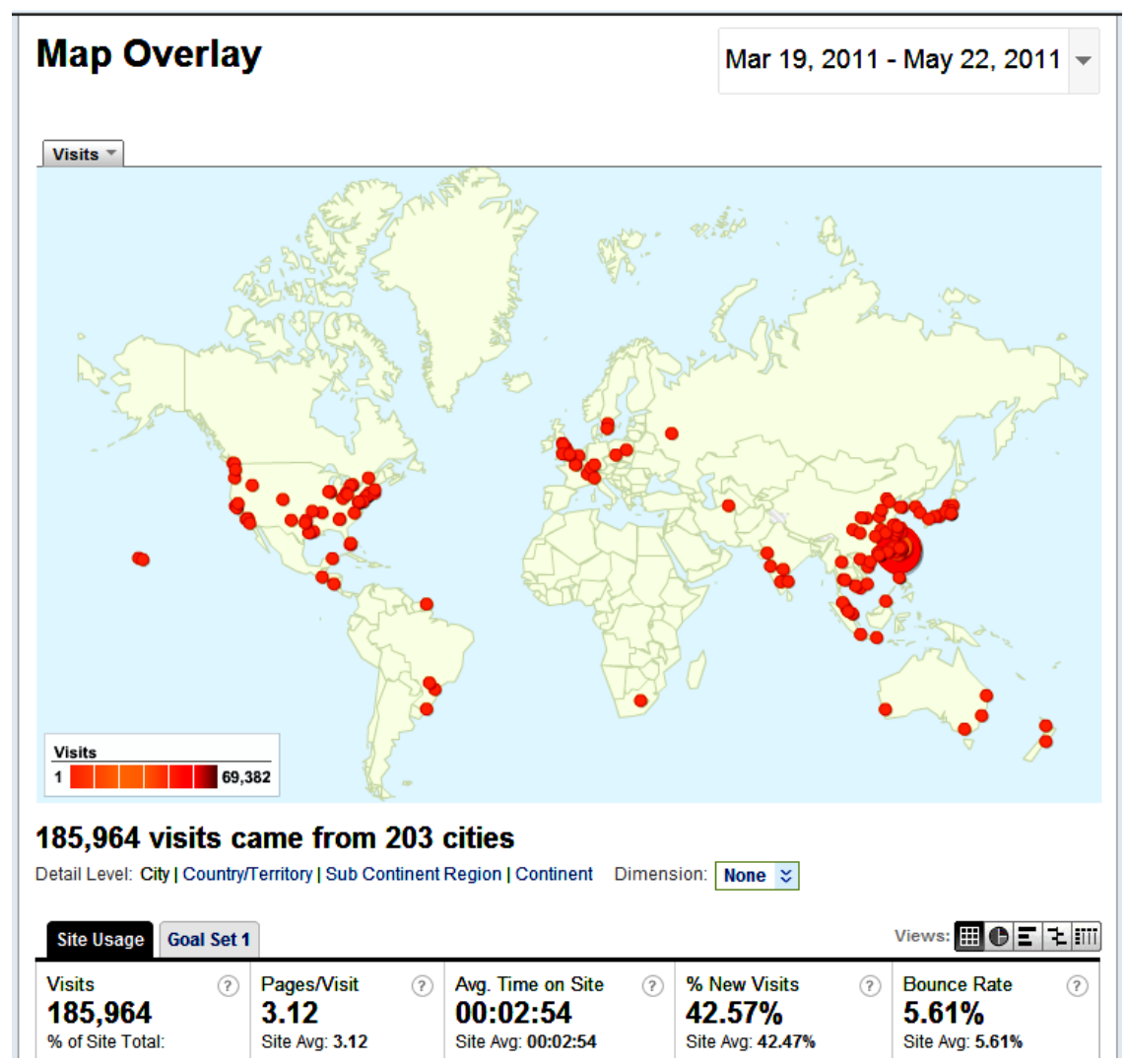

Figure 11. Geographic distribution of visitors by cities.

sands visits came from 200 different cities, mostly in Asia, North America, and Europe.

\subsection{Error Analysis}

GPS are still subject to satellites errors, tropospheric delay, ionospheric delay and other factors, and therefore its accuracy is merely 10 to 25 meters. In addition, GPS signals are highly affected by vigorous vibrations, especially when believers set off firecrackers while the palanquin passes by. The vibration of the palanquin would often result in abnormal reporting of coordinates by the GPS receiver (see Figure 12).

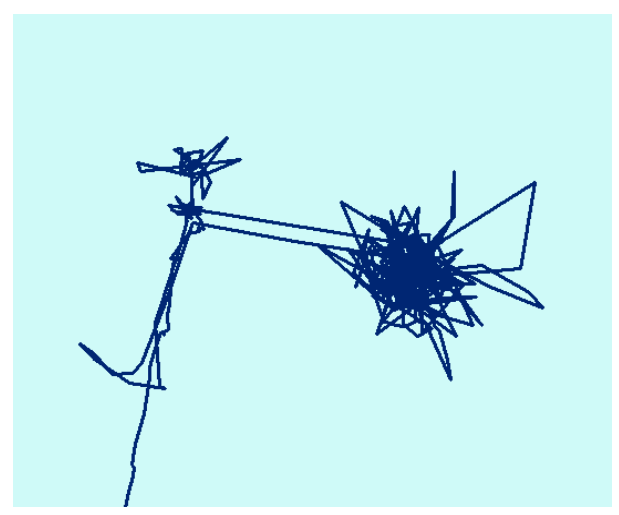

Figure 12. Abnormal GPS coordinates.
These abnormal coordinates need to be removed manually (see figure $\mathbf{1 3}$ for the outliers with a value higher than 200 meters). Therefore, strengthening the stability of hardware and developing an algorithm to automatically filter erroneous coordinates, such as to reduce the manual work and increase timeliness and reliability of information.

\section{Conclusions}

This article describes how to apply spatial technologies to Dajia Mazu Patrol and Pilgrimage, including the positioning

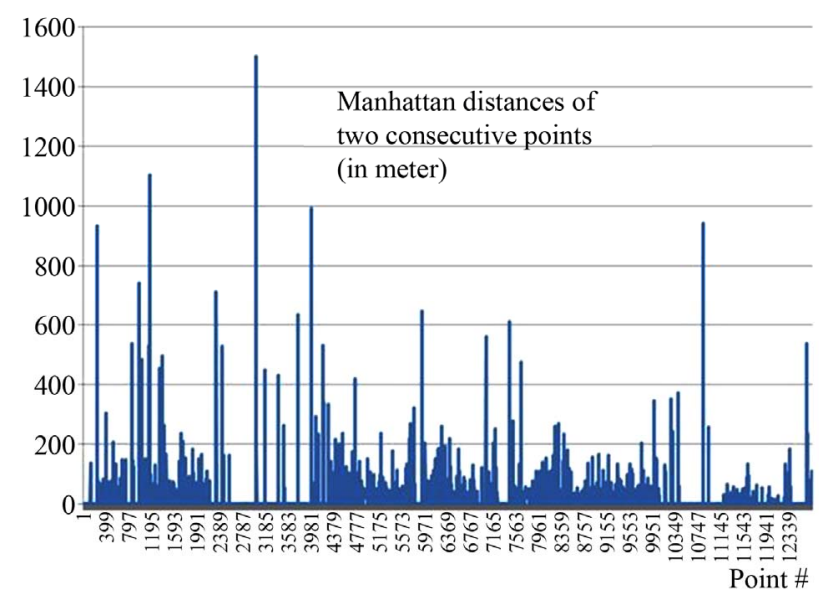

Figure 13. Analysis of abnormal coordinates. 
technology, equipment and operation framework adopted by the initiative. It also explores how spatial technologies can help organize the large-scale event. Publishing the real-time position of Mazu's palanquin on the internet can prevent pilgrims and tourists from tortures of long waiting. The historic trajectories can also assist festival organizers in choice of pilgrimage routes, and re-examine the planning in hindsight to improve future festival [10]. The following paragraphs summarize conclusions and future studies for public reference.

- This study successfully applied spatial technology and wireless communication to publish real-time positions of Mazu's palanquin and live video of pilgrimage on the internet, facilitating the festival proceeding. We demonstrated that technologies can boost the tourism boom, which could be a fusion paradigm among culture, tourism, and technology.

- During the patrol and pilgrimage, both battery of car kit and memory card of DVRs need to be replaced once every 12 hours. The system should solve the battery and memory card problems to reduce replacement frequency of equipment and thus the number of data loss.

The current system refreshes the video once 20 seconds with JPG images of $180 \times 131$ pixels. There are still significant deficiencies on image's clarity to be improved in the future.

\section{REFERENCES}

[1] V. Huang, "Journey of Devotion," Taiwan Review, Vol. 60 , No. 8, 2010.

[2] H. Hsun, "Happy Birthday Mazu," Proceedings of the Annual Meeting of Association for Asian Studies, Chicago, 2009, pp. 43-46.

[3] H. Hsun, "Incense-Offering and Obtaining the Magical Power of Chi': The Matsu (Heavenly Mother) Pilgrimage in Taiwan," Ph.D. Thesis, University of California, Ber- keley, 1993, pp. 1-10.

[4] C. Lin, "Aesthetics, Power and Consumption: Research on Warship Activities during Annual Festival of Dajia Matsu," Master's Thesis, Nanhua University, Chiayi City, 2005.

[5] H. Hsun, "Pilgrimage and Identity: Dajia Migrants and the Dajia Matsu Pilgrimage," Proceedings of the 48th Annual Meeting of the Association for the Asian Studies, Hawaii, 1996, pp. 28-32.

[6] E Chipman, "The Local Production of Culture in Beigan," Taiwan Journal of Anthropology, Vol. 6, No. 1, 2008, pp. 1-30.

[7] S. Tan, "The Cult and Festival of the Goddess of the Sea: A Maiden Encounter with Mazu," The Heritage Journal, Vol. 1, No. 1, 2004, pp. 13-20.

[8] S. Chung, "Gangster and Palanquin-Robbing in Mazu Pilgrimage Procession in Zhanghua, Taiwan," Master's Thesis, Nanhua University, Chiayi City, 2009.

[9] D. McCarthy, "Innovative Methods for Documenting Cultural Resources: Integrating GIS and GPS Technologies," CRM Journal, Vol. 1, No. 2, 2004, pp. 86-91.

[10] J. Lu, "The Study on Developmental Model of Dajia Mazu Culture Creative Industries," Master's Thesis, University of Kang Ning, Tainan City, 2009.

[11] P. Sangren, "Chinese Sociologics: An Anthropological Account of the Role of Alienation in Social Reproduction," Athlone Press, London, 2000, pp. 55-59.

[12] D. Huang, "On the Rites of Pilgrimage: A Case Study of Dajia Mazu," Journal of Chinese Ritual, Theatre, and Folklore, No. 158, 2007, pp. 9-38.

[13] H, Hsun, "Between Religion and State: Dajia Pilgrimage in Taiwan," Proceedings of Les Pelerinages: Parcoure Historiques, Parcours Croyants, Parcours Geographiques, Provence, 2008, pp. 16-20.

[14] M. Painho, M. Peixoto, P. Cabral and R. Sena, "WebGIS as a Teaching Tool," Proceedings of the ESRI User Conference, San Diego, 9-13 July 2001. 